\title{
Control of Oxide Particle Size by Oxygen Pressure for Internally Oxidized Silver Base Alloys*
}

\author{
By Mitsunori Sato**
}

\begin{abstract}
Silver base alloys containing $11.3 \mathrm{Cd}, 11.4 \mathrm{Cd}-0.24 \mathrm{Ni}, 7.62 \mathrm{Zn}-0.45 \mathrm{Te}, 6.13 \mathrm{Zn}-0.97 \mathrm{Sn}-0.48 \mathrm{Te}$, and $6.05 \mathrm{Zn}-1.03 \mathrm{Sn}-0.99 \mathrm{In}-0.47 \mathrm{Te}$ (at \%) have internally been oxidized at 1023, 1073 and $1123 \mathrm{~K}$ under continuously changing pressure of oxygen and in air. The size of oxide particles has been controlled by oxygen pressure.

(1) In order to disperse the same size of oxide particles in all internal oxidation layers, the oxygen pressure $P$ should be controlled with time $t$ as $P=K^{4} P_{\mathrm{a}}^{2} t^{2} / 4 X_{\mathrm{a}}^{4}$, where $K$ is the velocity constant of internal oxidation at a constant oxygen pressure $P_{a}$, and $X_{a}$ is the distance from the surface. The value of $K$ depends on the composition of specimens and the temperature of internal oxidation. (2) The activation energies for internal oxidation of the alloys are smaller than $92 \mathrm{~kJ} / \mathrm{mol}$ estimated from the diffusion of oxygen in silver. (3) The diffusivity of oxygen in internal oxidation layers and its activation energy are evaluated to be smaller than those in silver.
\end{abstract}

(Received March 12, 1982)

\section{Introduction}

The internal oxidation process has been applied widely to the production of dispersion hardening materials or electrical contact materials. The dispersed structure of oxide particles precipitated in a silver matrix depends on the composition of specimens and the conditions of internal oxidation: The size of the oxide particles decreases with decreasing solute concentration and internal oxidation temperature, and decreases with increasing oxygen pressure. Moreover, it is well known that the size increases as the internal oxidation proceeds to inner direction ${ }^{(1)-(11)}$. Some characteristics of internally oxidized alloys depend on the composition of specimens and the dispersed structure of oxide particles ${ }^{(5)(12)-(14)}$. Especially, for electrical contact materials with dispersed structures different from position to position in internal oxidation layers, the resistance to welding adhesion changes as the erosion progresses by switching operations ${ }^{(15)}$. If a uniform dispersed structure is obtained in all the internal oxidation layers, the characteristics of electrical contacts are stahle and

* This paper was originally published in Japanese in J. Japan Inst. Metals, 44 (1980), 567.

** National Research Institute for Metals, 2-1 Sengen-1, Sakura-mura, Niihari-gun, Ibaraki 305, Japan. the reliability is improved. In order to disperse the same size of oxide particles in all the layers, it must be considered to control the oxygen pressure or the temperature in the internal oxidation process $^{(2)-(4)(6)(7)(10)}$.

In the present work, therefore, we investigated the internal oxidation behavior for $\mathrm{Ag}-\mathrm{Cd}$ and $\mathrm{Ag}-\mathrm{Zn}$ base alloys used as electrical contact materials, and tried to control the size of oxide particles by continuously changing pressure of oxygen in the internal oxidation process.

\section{Experimental}

Table 1 shows the chemical compositions of specimens used. The specimens were prepared in a size of $10 \times 10 \times 10 \mathrm{~mm}$ after melting 0.6 $\mathrm{kg}$ of components from $\mathrm{Ag}$ of $99.9 \%, \mathrm{Cd}$ of $99.9 \%, \mathrm{Ni}$ of $99.9 \%, \mathrm{Zn}$ of $99.8 \%$, Sn of $99.9 \%$, In of $99.999 \%$ and $\mathrm{Te}$ of $99.99 \%$, and were internally oxidized in continuously changing

Table 1 Chemical compositions of specimens (at $\%$ ).

\begin{tabular}{lccccccc}
\hline $\begin{array}{c}\text { Speci- } \\
\text { men }\end{array}$ & Cd & Ni & Zn & Sn & In & Te & Ag \\
\hline CdO & 11.3 & - & - & - & - & - & Bal \\
CdON & 11.4 & 0.24 & - & - & - & - & Bal \\
$800 T$ & - & - & 7.62 & - & - & 0.45 & Bal \\
$610 T$ & - & - & 6.13 & 0.97 & - & 0.48 & Bal \\
$611 T$ & - & - & 6.05 & 1.03 & 0.99 & 0.47 & Bal \\
\hline
\end{tabular}


pressure of oxygen and in air at 1023, 1073 and $1123 \mathrm{~K}$.

The dispersed structures of oxide particles were observed using an optical microscope.

\section{Results}

Photograph 1 shows the microstructures of the specimens internally oxidized in air at 1073 and $1123 \mathrm{~K}$. The size of oxide particles is found to increase as the internal oxidation proceeds to inner direction and as the temperature increases. The dispersed structures depend on the composition of specimens, and further, on the stability of oxides and the diffusivity of solute elements ${ }^{(6)(12)(16)}$.

Figure 1 shows the relation between the thickness $X$ of the layer internally oxidized in

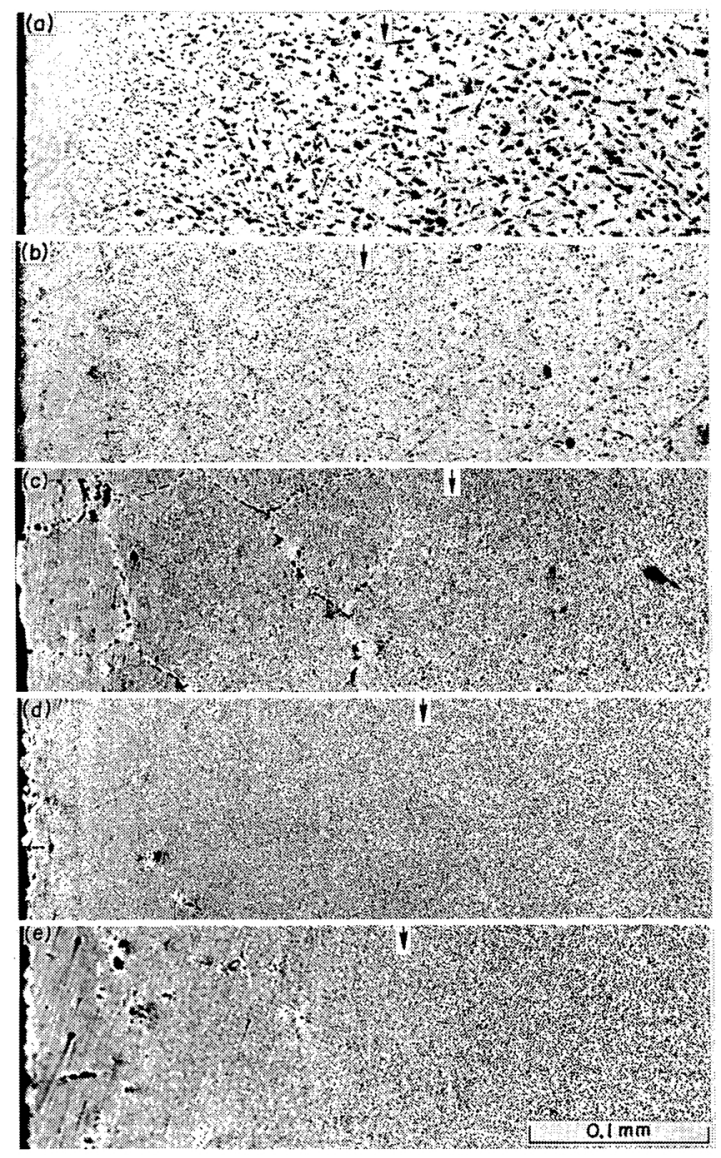

(A) air and the time $t$, where (a) is oxidized at $1023 \mathrm{~K}$, (b) at $1073 \mathrm{~K}$, and (c) at $1123 \mathrm{~K}$. The thickness $X$ is proportional to $t^{1 / 2}$.

Now, we will consider how the size of oxide particles is controlled by the oxygen pressure in the internal oxidation process. The thickness $X$ has been given by Wagner ${ }^{(1)}$ as

$$
X=\left(\frac{2 N_{0} D_{0} t}{v N_{m}}\right)^{1 / 2},
$$

where $N_{0}$ is the equilibrium solubility of oxygen in the solvent metal for the given oxidizing atmosphere, $N_{m}$ is the mole fraction of the solute element in the alloy before internal oxidation, $D_{0}$ is the diffusivity of oxygen in the internal oxidation layer, $v$ is the ratio in number of oxygen atoms to solute atoms of the precipitated oxide, and $t$ is the oxidation time.

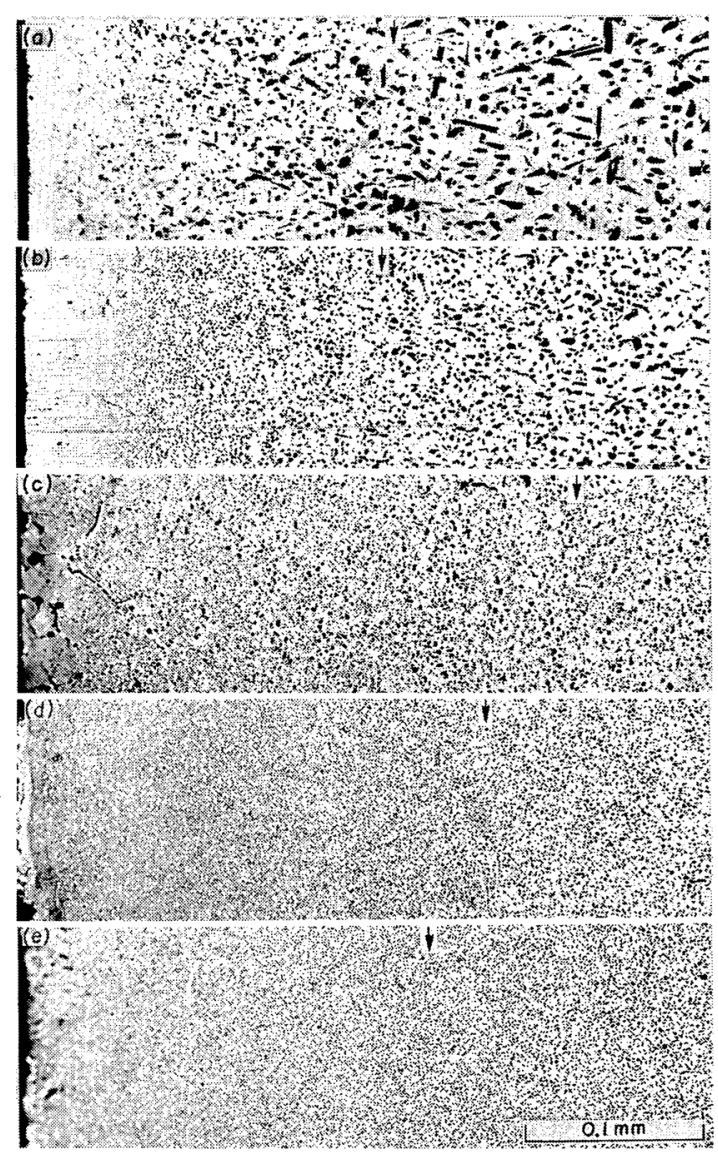

(B)

Photo. 1 Microstructures of specimens internally oxidized in air. (a) CdO, (b) CdON, (c) 800T, (d) $610 \mathrm{~T}$, (e) $611 \mathrm{~T}$. (A) Internally oxidized at $1073 \mathrm{~K}$. (B) Internally oxidized at $1123 \mathrm{~K}$. 

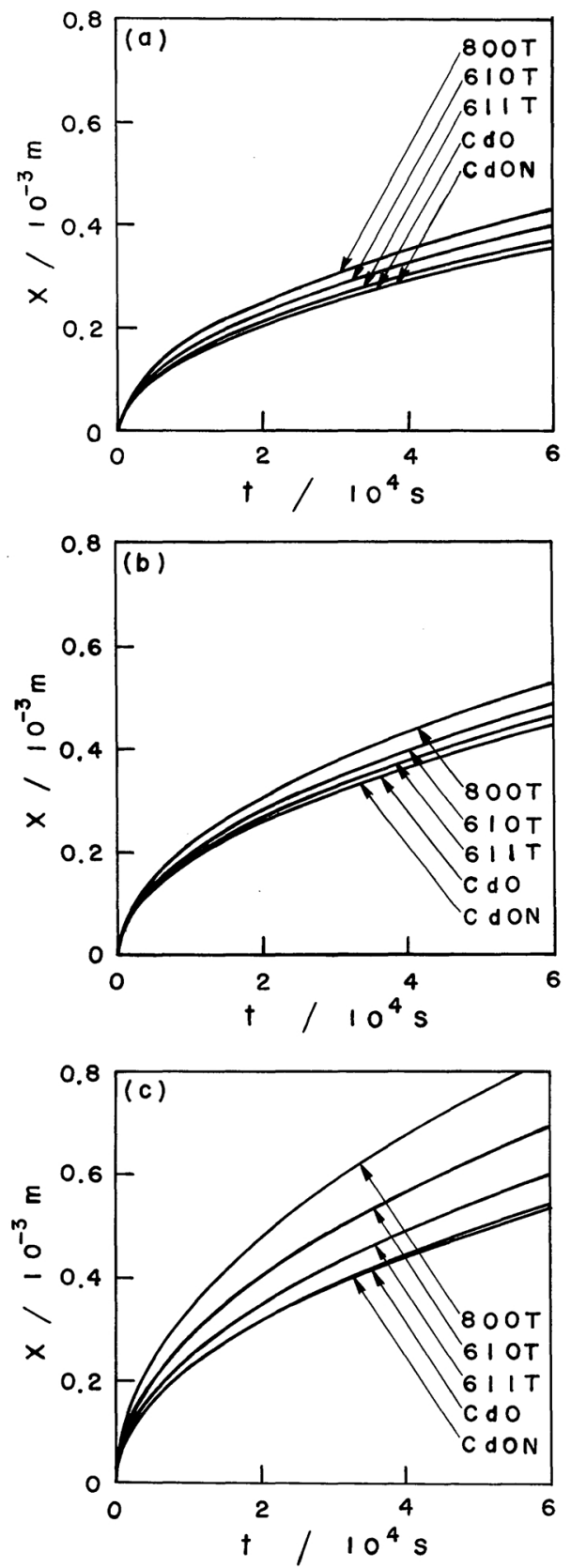

Fig. 1 Relation between layer thickness $X$ internally oxidized in air and time $t$. (a) Internally oxidized at $1023 \mathrm{~K}$. (b) Internally oxidized at $1073 \mathrm{~K}$. (c) Internally oxidized at $1123 \mathrm{~K}$.

When the composition of specimen and the temperature of internal oxidation are fixed,

$$
\left(\frac{2 D_{0}}{v N_{m}}\right)^{1 / 2}=K_{1}
$$

where $K_{1}$ is a constant. Equation (1) can be rewritten as

$$
X=K_{1}\left(N_{0} t\right)^{1 / 2} .
$$

When the gradient of oxygen concentration in the oxidation layer decreases, the diffusion range of solute element increases, because the supply of oxygen to the internal oxidation front decreases. The size of oxide particles increases, therefore, as the internal oxidation proceeds to inner direction. Namely, the size may have a strong dependence on the ratio of the number of the solute atoms to that of oxygen atoms supplied to the internal oxidation front ${ }^{(6)(7)}$. When the ratio is constant, the internal oxidation velocity $X^{\prime}$ is constant. In this case the uniform size of oxide particles will be controlled in all the layers. From Eq. (3) when

$$
N_{0} \propto t \text {, }
$$

$X^{\prime}$ is constant. Silver has sufficiently high oxygen solubility, in this case, the relation between the oxygen pressure $P$ and $N_{0}$ is given by

$$
N_{0}=K_{2} P^{1 / 2} \text {. }
$$

The thickness $X$ is given by ${ }^{(6)}$

$$
X=K P^{1 / 4} t^{1 / 2} \text {. }
$$

In order to obtain the same size of oxide particles as that at $X=X_{a}$ when the internal oxidation proceeds in the constant pressure $P_{a}$ of oxygen and at a constant temperature, the value of $X^{\prime}$ is given from the differentiation of Eq. (6) by $t$ as

$$
X^{\prime}=\frac{K P_{a}^{1 / 4}}{2 t_{a}^{1 / 2}},
$$

and the thickness $X$ must be in proportion to the time $t$ as

$$
X=X^{\prime} t \text {. }
$$

The constant $K$ is obtained from substituting the layer thickness $X_{b}$, formed by internal oxidation during the time $t_{b}$, into Eq. (6) as

$$
K=\frac{X_{b}}{P^{1 / 4} t_{b}^{1 / 2}} .
$$

Table 2 shows the values of $K$ obtained from substituting the results of Fig. 1 into Eq. (9), 
Table 2 Velocity constant $K$ of internal oxidation in air.

\begin{tabular}{lrrr}
\hline Specimen & \multicolumn{3}{c}{$K$} \\
\cline { 2 - 4 } & $1023 \mathrm{~K}$ & $1073 \mathrm{~K}$ & $1123 \mathrm{~K}$ \\
\hline CdO & $1.21 \times 10^{-7}$ & $1.50 \times 10^{-7}$ & $1.84 \times 10^{-7}$ \\
CdON & $1.20 \times 10^{-7}$ & $1.49 \times 10^{-7}$ & $1.82 \times 10^{-7}$ \\
$800 \mathrm{~T}$ & $1.46 \times 10^{-7}$ & $1.79 \times 10^{-7}$ & $2.80 \times 10^{-7}$ \\
$610 \mathrm{~T}$ & $1.36 \times 10^{-7}$ & $1.65 \times 10^{-7}$ & $2.35 \times 10^{-7}$ \\
$611 \mathrm{~T}$ & $1.26 \times 10^{-7}$ & $1.57 \times 10^{-7}$ & $2.03 \times 10^{-7}$ \\
\hline
\end{tabular}

where the units of $X_{b}, P$ and $t_{b}$ are $\mathrm{m}, \mathrm{Pa}$ and $\mathrm{s}$, respectively. The time $t_{a}$ during which the internal oxidation proceeds from the surface to $X_{a}$ is given from Eq. (6) as

$$
t_{a}=\frac{X_{a}^{2}}{K^{2} P_{a}^{1 / 2}} .
$$

When the oxygen pressure $P$ changes with the time $t$ as

$$
P=K_{a} t^{2},
$$

Equation (6) is represented by Eq. (8). The constant $K_{a}$ can be obtained as follows: In Eq. (8) the time $t_{c}$ corresponding to $X=X_{a}$ is given by

$$
t_{c}=\frac{X_{a}}{X^{\prime}}
$$

Since $P$ reaches $P_{a}$ at $t=t_{c}$, the constant $K_{a}$ is given by

$$
K_{a}=\frac{P_{a}}{t_{c}^{2}} .
$$

The oxygen pressure $P$ is obtained from Eqs. (7), (9), (10), (11), (12) and (13) as

$$
P=\frac{K^{4} P_{a}^{2}}{4 X_{a}^{4}} t^{2}=\frac{P_{a} X_{b}^{4}}{4 X_{a}^{4} t_{b}^{2}} t^{2} .
$$

When $P$ changes with $t$ following Eq. (14), the dispersed structure with the same size of oxide particles as that at $X_{a}$ may be controlled in the whole layer.

Moreover, when $K_{a}$ is arbitrary in Eq. (11), $X^{\prime}$ and $X_{a}$ are obtained from Eqs. (7), (10), (12) and (13) as

$$
X^{\prime}=\frac{K K_{a}^{1 / 4}}{2^{1 / 2}},
$$

and

$$
X_{a}=\frac{K P_{a}^{1 / 2}}{2^{1 / 2} K_{a}^{1 / 4}} .
$$

Figure 2 shows the condition under which the dispersed structure with the same size of oxide particles as that of each position shown with arrows in Photo. 1 is controlled in the whole layers. Figure 2(a) shows the relation

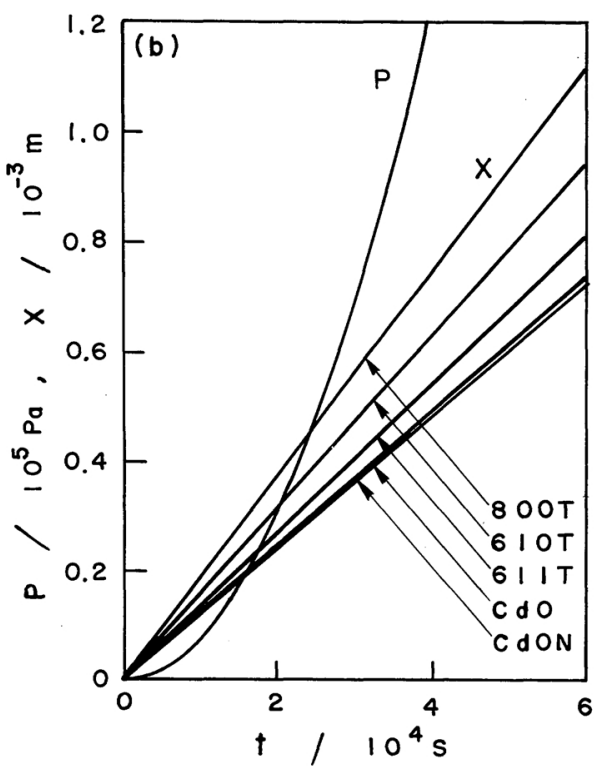

Fig. 2 Relations between oxygen pressure $P$, layer thickness $X$ and time $t$ of internal oxidation. (a) Internal oxidation temperature $1073 \mathrm{~K}$. (b) Internal oxidation temperature $1123 \mathrm{~K}$. 
between the oxygen pressure $P$ and the time $t$ obtained from Eq. (14), and the relation between the layer thickness $X$ and the time $t$ obtained from Eqs. (8) and (15), in the case of internal oxidation at $1073 \mathrm{~K}$ under an oxygen pressure of $2 \times 10^{4} \mathrm{~Pa}$ (in air). The positions of $X_{a}$ are $0.200 \mathrm{~mm}$ for the specimen $\mathrm{CdO}, 0.199 \mathrm{~mm}$ for $\mathrm{CdON}, 0.238 \mathrm{~mm}$ for $800 \mathrm{~T}, 0.220 \mathrm{~mm}$ for $610 \mathrm{~T}$ and $0.208 \mathrm{~mm}$ for $611 \mathrm{~T}$, as shown with arrows in Photo. 1(A). Figure 2(b) shows the case of internal oxidation at $1123 \mathrm{~K}$. The positions $X_{a}$ are $0.200 \mathrm{~mm}$ for $\mathrm{CdO}, 0.198 \mathrm{~mm}$ for $\mathrm{CdON}, 0.305 \mathrm{~mm}$ for $800 \mathrm{~T}, 0.256 \mathrm{~mm}$ for $610 \mathrm{~T}$ and $0.221 \mathrm{~mm}$ for $611 \mathrm{~T}$, as shown with arrows in Photo. 1(B).

Photograph 2 shows the microstructures of

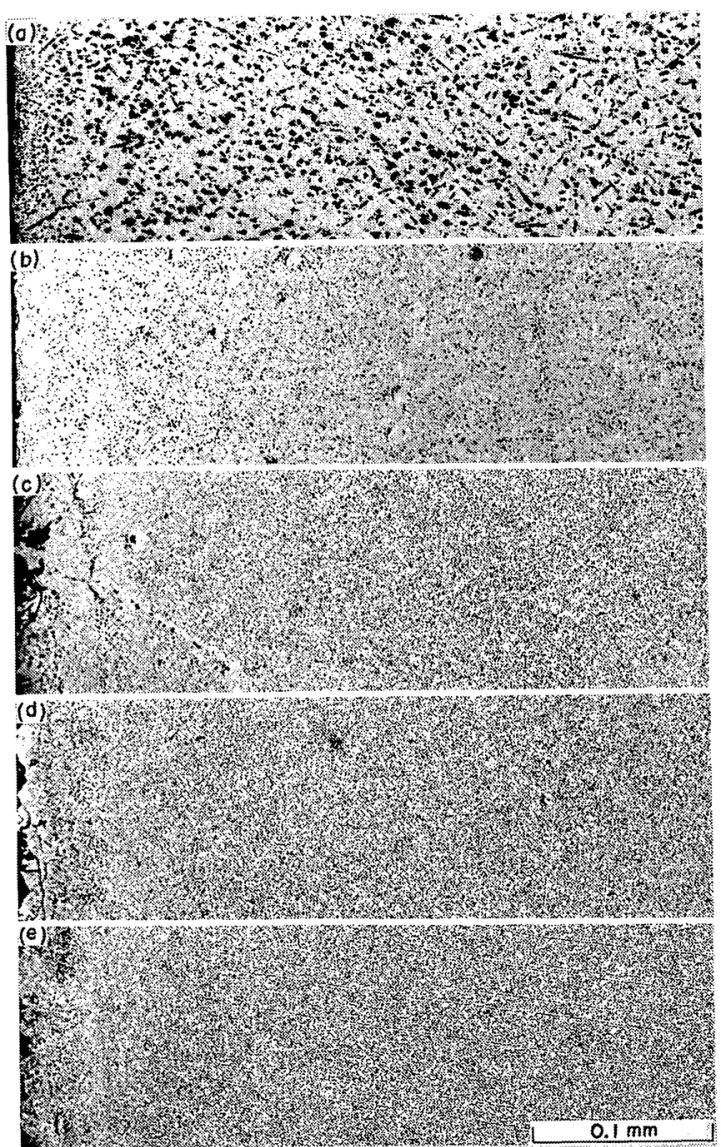

(A) the internally oxidized specimens when the oxygen pressure is changed continuously, as shown in Fig. 2. The same size of oxide particles as that shown with arrows in Photo. 1 is found to be controlled, except for a thin layer about $10 \mu \mathrm{m}$ distant from the surface. In the thin layer the oxide particles are fine. It is known that near the surface, i.e. at the place about $10 \mu \mathrm{m}$ distant from it, the diffusion process is complicated and different from the diffusion in a solid ${ }^{(16)}$. Moreover, $\mathrm{Cd}$ and $\mathrm{Zn}$ in the alloys are evaporated selectively from the surface before oxygen is supplied, after the temperature of specimens has reached the equilibrium, because the vapor pressure of the elements is high. Therefore, near the surface the solute concentration will decrease.
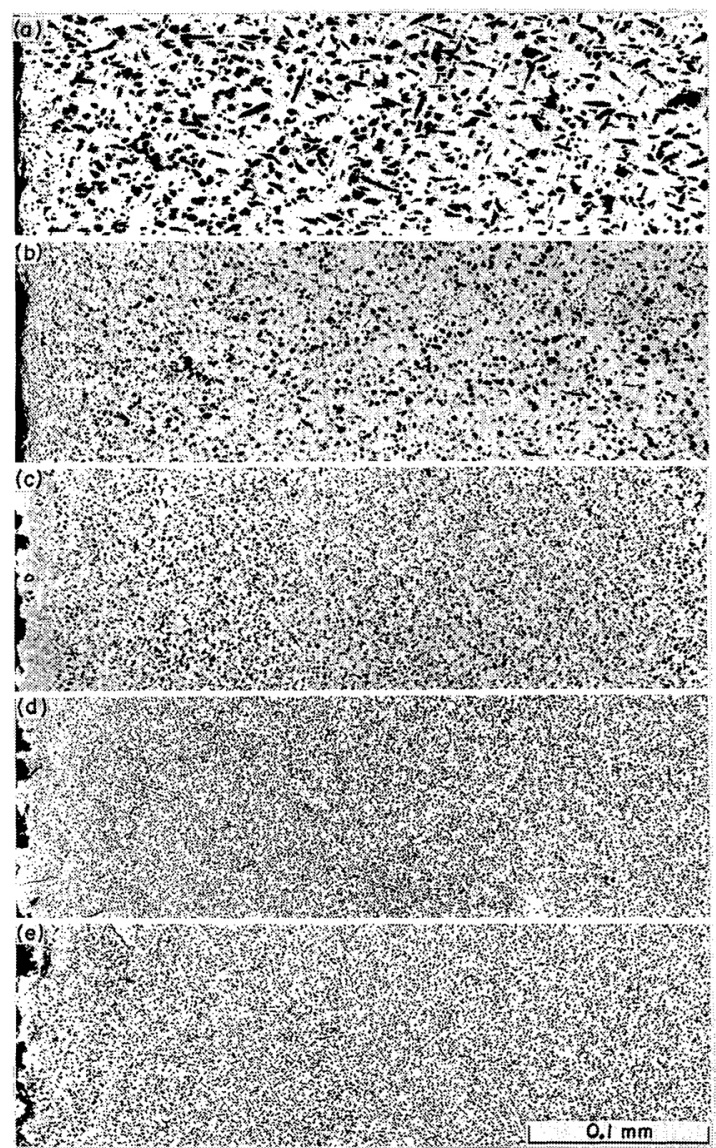

(B)

Photo. 2 Microstructures of specimens internally oxidized at controlled oxygen pressure as shown in Fig. 2. (a) CdO, (b) CdON, (c) 800T, (d) 610T, (e) 611T. (A) Internally oxidized at $1073 \mathrm{~K}$. (B) Internally oxidized at $1123 \mathrm{~K}$. 


\section{Discussion}

We will examine the activation energy for internal oxidation, and the diffusivity of oxygen and its activation energy in the internal oxidation layer in which the oxide particles are dispersed in the silver matrix. When the internal oxidation proceeds under the constant pressure of oxygen, $\left(K P_{a}^{1 / 4}\right)^{2}=K_{0}$, Eq. (6) can be rewritten as

$$
X^{2}=K_{0} t .
$$

Figure 3 shows the relation between the constant $K_{0}$ obtained from $K$ in Table 2 and the reciprocal of temperature $T^{-1}$. Table 3 shows the activation energy obtained from the gradient of each line using the Arrhenius equation. Here, the values of $K_{0}$ for specimens $800 \mathrm{~T}$, $610 \mathrm{~T}$ and $611 \mathrm{~T}$ at $1123 \mathrm{~K}$ were excepted

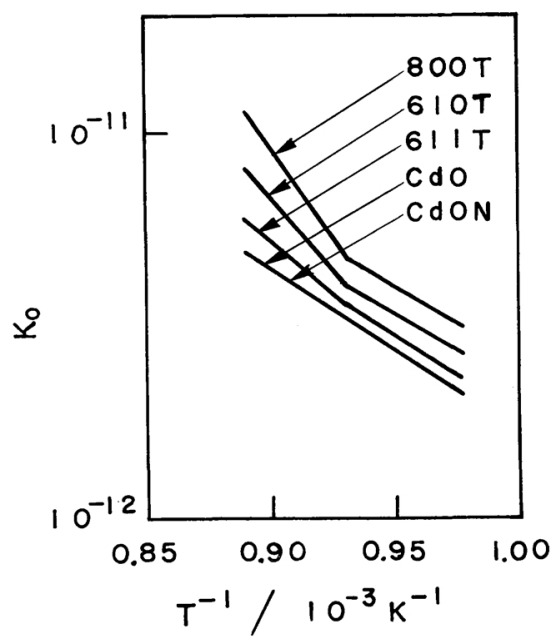

Fig. 3 Relation between velocity constant $K_{0}$ of internal oxidation in air and reciprocal of temperature $T^{-1}$.

Table 3 Activation energy $Q$ obtained from $K_{0}$.

\begin{tabular}{cc}
\hline \hline Specimen & $Q / 10^{3} \mathrm{~J} \cdot \mathrm{mol}^{-1}$ \\
\hline CdO & 78 \\
CdON & 81 \\
$800 \mathrm{~T}$ & 75 \\
$610 \mathrm{~T}$ & 71 \\
$611 \mathrm{~T}$ & 80 \\
$\mathrm{Ag}$ & $92^{*}$ \\
\hline
\end{tabular}

* after Eichenauer and Müller( ${ }^{(2)}$. because the temperature approaches the liquidus and $K_{0}$ increases abruptly. The values of $K_{0}$ are also obtained from Eqs. (1) and (17) as

$$
K_{0}=\frac{2 N_{0} D_{0}}{v N_{m}},
$$

where the values of $v$ and $N_{m}$ are determined from the composition of specimens. Therefore, the activation energy obtained from the experimental value of $K_{0}$ may agree with that obtained from $N_{0} D_{0}$. The activation energies for all the specimens are smaller than $92 \mathrm{~kJ} /$ mol which was obtained from $N_{0} D_{0}$ of oxygen in silver by Eichenauer et al. ${ }^{(2)(4)}$. Moreover, we will compare the value of $D_{0}$ of oxygen in the internal oxidation layers in which the oxide particles are dispersed in the silver matrix. The $D_{0}$ in silver has been given by Eichenauer et al. ${ }^{(2)(4)}$ as

$$
D_{0}=3.66 \times 10^{-7} \exp \left(\frac{-46030}{R T}\right) \mathrm{m}^{2} \mathrm{~s}^{-1} \text {. }
$$

On the other hand, in order to obtain $D_{0}$ from $N_{0} D_{0}$ of oxygen in the internal oxidation layers obtained experimentally, the value of $N_{0}$ must be known at each temperature. The value of $N_{0}$ in the layers is approximated by that in silver $^{(2)(4)(9)}$, namely,

$$
N_{0}=6.69 \times 10^{-2} \exp \left(\frac{-49620}{R T}\right),
$$

at $P=10^{5} \mathrm{~Pa}$.

Table 4 shows the values of $D_{0}$ obtained from Eqs. (5), (18), (20) and the values of $K_{0}$ obtained from $K$ in Table 2. The value of $D_{0}$ for each specimen is smaller than that for silver obtained from Eq. (19). It has been reported ${ }^{(5)(7)}$

Table 4 Diffusivity $D_{0}$ of oxygen in internal oxidation layers.

\begin{tabular}{lccc}
\hline & \multicolumn{3}{c}{$D_{0} / 10^{-9} \mathrm{~m}^{2} \mathrm{~s}^{-1}$} \\
\cline { 2 - 4 } & $1023 \mathrm{~K}$ & $1073 \mathrm{~K}$ & $1123 \mathrm{~K}$ \\
\hline CdO & 1.33 & 1.57 & 1.83 \\
CdON & 1.36 & 1.60 & 1.85 \\
$800 \mathrm{~T}$ & 1.47 & 1.68 & - \\
$610 \mathrm{~T}$ & 1.35 & 1.51 & - \\
$611 \mathrm{~T}$ & 1.35 & 1.59 & - \\
$\mathrm{Ag}$ & $1.63^{*}$ & $2.10^{*}$ & $2.65^{*}$ \\
\hline \hline
\end{tabular}

* after Eichenauer and Müller(2). 
Table 5 Activation energy $Q$ obtained from $D_{0}$.

\begin{tabular}{cc}
\hline Specimen & $Q / 10^{3} \mathrm{~J} \cdot \mathrm{mol}^{-1}$ \\
\hline CdO & 31 \\
CdON & 31 \\
$800 \mathrm{~T}$ & 26 \\
$610 \mathrm{~T}$ & 22 \\
$611 \mathrm{~T}$ & 31 \\
$\mathrm{Ag}$ & $46^{*}$ \\
\hline \hline
\end{tabular}

* After Eichenauer and Müller(2).

that $D_{0}$ in the internal oxidation layers changes when the diffusion occurs in the interface between oxide particles and matrix, and when the cross-sectional area to the diffusion of oxygen decreases with dispersion of oxide particles. Therefore, the value of $D_{0}$ depends on the size and the density of oxide particles. In such dispersed structures as shown in Photo. 1 , the decrease in $D_{0}$ due to the decrease in the cross-sectional area may overcome the increase in $D_{0}$ due to the diffusion in the interface, because the particle size is large. Table 5 shows the activation energy obtained from $D_{0}$ in Table 4 . The activation energies of $D_{0}$ for specimens are smaller than $46 \mathrm{~kJ} / \mathrm{mol}$ in Eq. (19) for silver. The activation energy of the diffusion in the interface is smaller than that of the diffusion in solid. However, since from the value of $D_{0}$ examined above the dependence of the diffusion in the interface is considered to be small, the small activation energy of $D_{0}$ in the internal oxidation layers can not be explained only by the existence of oxide particles. The size of oxide particles decreases and its density increases with decreasing internal oxidation temperature, as found in Photos. 1 and 2. Therefore, the ratio of $D_{0}$ in the internal oxidation layers to $D_{0}$ in silver may become high because the effect of the diffusion in the interface increases with decreasing internal oxidation temperature. Oxygen diffuses from the surface in the direction of the internal oxidation front, and on the other hand, the solute element diffuses from the unoxidized region to the front. Therefore, when the whole area of specimen is internally oxidized, a zone deficient in oxide particles appears in the center of specimen. It has been reported ${ }^{(6)}$ that the width of the depleted zone is inversely proportional to the square root of the diffusion velocity ratio of solute element to oxygen and this ratio changes with the internal oxidation temperature, and the width increases with increasing temperature. Namely, when a sufficiently thick specimen is internally oxidized, the value of $N_{m}$ in Eq. (1) may increase up to that of the original alloy with increasing temperature. Since the value of $D_{0}$ was obtained from the value of $N_{m}$ for the original alloy, the ratio of $D_{0}$ in the internal oxidation layers to $D_{0}$ in silver decreases with increasing temperature. Moreover, in Eq. (1) the value of $v$ was used for the stoichiometric oxide, but the precipitated oxide is in an oxygen poor type. Since the degree of oxygen poorness is affected by the temperature, the value of $D_{0}$ and its gradient change. Since these factors are multiplied together, the activation energy of the diffusion of oxygen in the internal oxidation layers may be smaller than the real value.

When the internal oxidation proceeds in continuously changing pressure of oxygen at a constant temperature, the value of $N_{0}$ in Eq. (1) changes. Therefore, the condition controlling the dispersed structure is determined easily. On the other hand, in order to control the dispersed structure by the temperature, the temperature must decrease as the internal oxidation proceeds inwardly from the surface. This means a decrease in the value of $K_{0}$ in Eq. (17) with increasing time $t$ (layer thickness $X)$. The oxidation velocity extremely decreases as the internal oxidation proceeds inwards. Moreover, when silver base alloys are internally oxidized under a constant pressure of oxygen, the size of oxide particles in a layer $100 \mu \mathrm{m}$ distant from the surface is much smaller than that in the inside, as found in Photo. 1. In order to control the size of oxide particles near the surface, the temperature must extremely increase. For silver base alloys the maximum temperature is about $1120 \mathrm{~K}$, limited from the melting temperature. Namely, it is very difficult to control the dispersed structure near the surface by the temperature.

\section{Summary}

The internal oxidation behavior in $\mathrm{Ag}-\mathrm{Cd}$ 
and $\mathrm{Ag}-\mathrm{Zn}$ base alloys was investigated, and it was tried to control the size of oxide particles under continuously changing pressure of oxygen.

(1) In order to disperse the same size of oxide particles in all the internal oxidation layers, the oxygen pressure $P$ should be controlled with the time $t$ as $P=K^{4} P_{a}^{2} t^{2} / 4 X_{a}^{4}$, where $K$ is the velocity constant of internal oxidation under a constant oxygen pressure $P_{a}$, and $X_{a}$ is the distance from the surface. The value of $K$ depends on the composition of specimens and the temperature of internal oxidation. (2) The activation energy for the internal oxidation obtained is smaller than $92 \mathrm{~kJ} / \mathrm{mol}$ estimated from the diffusion of oxygen in silver by Eichenauer and Müller. (3) The diffusivity of oxygen in internal oxidation layers and its activation energy are evaluated to be smaller than those in silver.

\section{REFERENCES}

(1) C. Wagner: Z. Electrochem., 63 (1959), 772.

(2) W. Eichenauer and G. Müller: Z. Metallk., 53 (1962), 321.
(3) J. S. Wolf and E. B. Evans: Corrosion, 18 (1962), 129.

(4) J. E. Verfurth and R. A. Rapp: Trans. Met. Soc. AIME, 230 (1964), 1310.

(5) S. Goto and S. Koda: J. Japan Inst. Metals, 32 (1968), 334 (in Japanese).

(6) S. Kabayama and E. Kamijo: J. Japan Inst. Metals, 32 (1968), 1204 (in Japanese).

(7) S. Goto and S. Koda: J. Japan Inst. Metals, 34 (1970), 319 (in Japanese).

(8) K. Saito and T. Hikage: J. Japan Inst. Metals, 40 (1976), 227 (in Japanese).

(9) O. Michikami and S. Yamamoto: J. Japan Inst. Metals, 41 (1977), 551 (in Japanese).

(10) K. Hauffe: Oxidation of Metals, Plenum Press, New York, (1965), p. 335.

(11) C. R. Cupp: Progr. Metal. Phys., London, 4 (1953), 105.

(12) H. Kimata and S. Nishi: J. Japan Inst. Metals, 35 (1971), 615 (in Japanese).

(13) K. Suzuki, T. Okutomi and S. Mito: J. Japan Inst. Metals, 41 (1977), 80 (in Japanese).

(14) O. Michikami: J. Japan Inst. Metals, 42 (1978), 484 (in Japanese).

(15) I. Morimoto, M. Sato and M. Hijikata: J. Japan Inst. Metals, 32 (1968), 597 (in Japanese).

(16) M. Sugiyama: Kinzoku-zairyo no Kanetsu to Sanka (Heating and Oxidation of Metals), Seibundo Shinkosha (1955), p. 52. 